\title{
Clusters outskirts at X-ray wavelengths: current status and future prospects
}

\author{
Silvano Molendi \\ Istituto di Astrofisica e Fisica Cosmica, Sezione di Milano, Via Bassini 15, I-20133, Milano, \\ Italy email: silvano@mi.iasf.cnr.it
}

\begin{abstract}
A solid observational characterization of cluster regions around the virial radius would allow us to improve considerably our understanding of the physics of galaxy clusters as a whole: sadly current and planned experiments will not allow us to study these regions. Unbeknownst to most but not all, the development of an experiment sensitive to cluster outer regions could be achieved with currently available technology, with no need for breakthroughs. Amongst the major factors that will decide whether and when such an experiment will be flown are the awareness of the cluster community of the importance of such a mission and its determination at supporting it.
\end{abstract}

\section{Why studying cluster outskirts?}

In the hierarchical Universe clusters form at the intersection of cosmic filaments through accretion (see for example the picture on the the conference poster taken from Borgani et al. 2004). Shocks are expected to occur where the free-falling gas collides with the ICM (e.g. Tozzi et al. 2000). These shocks convert the bulk of the kinetic energy of the freefalling gas into thermal energy, a fraction of the kinetic energy may be retained by the shocked gas (e.g. Schuecker et al. 2004), playing a non trivial role in shaping the cluster. The boundary between cluster and free falling gas is likely not in a spherical form, as accretion occurs mainly from a few preferential directions. The thermodynamic status of the pre-shocked gas will likely determine the strength of the shock; if, for example, the gas has been pre-shocked in filaments, as envisaged in the pre-heating scenario, the shock will be weaker than if it has not been pre-shocked (e.g. Tozzi et al. 2000).

Relatively massive in-falling substructures, such as groups, are likely to retain at least part of their structure all the way down to the cluster central regions (e.g. A3667, Vikhlinin et al. 2001). Shocks generated by these structures will likely leave turbulence in their wakes, which will eventually be dissipated at smaller scales (e.g. Sunyaev these proceedings, Schuecker et al. 2004). Less massive structures such as galaxies will suffer severe modifications already in the cluster outskirts. The compression associated to the shock will amplify the strength of the magnetic fields which are frozen in the high conductivity plasma, and particles will likely be accelerated at the site of the shock.

Ram pressure stripping of the metal rich gas from the in-falling galaxies by the ICM (e.g. Treu, Moore these proceedings) will enrich the ICM in heavy elements. Direct accretion of pristine gas, never processed in galaxies, will have the opposite effect of diluting the metal content of the ICM.

These simple considerations illustrate how important the regions around the virial radius, the cluster outskirts, are. The rather obvious reason for the occurrence of the wide variety of phenomena sketched above is that these are the regions where virialization occurs. Viewing the issue from an observer's point of view we may say that as long as we do not have a solid observational characterization of cluster outer regions our understanding of clusters as a whole will necessarily be a limited one. 


\section{How much do available observations tell us?}

Let us then see how much of this extremely important observational work we can carry out with currently available data. Let us consider the three principle X-ray observables: the surface brightness, the temperature and the metal abundance.

Measurements of the surface brightness extend out to about $0.6 \mathrm{r}_{180}$ (Mohr et al. 1999, Ettori \& Fabian 1999, Ettori et al. 2004). The detector that has been most successful in these measurements, at low redshifts, i.e. $z<0.2$, is not part of the payload of the latest generation of X-ray satellites, indeed it is the ROSAT PSPC, which was launched 14 year ago.

ASCA (Markevitch et al. 1998), BeppoSAX (De Grandi \& Molendi 2002) and XMMNewton (Pratt \& Arnaud 2002, Zhang et al. 2004) allow us to extend temperature measurements out to about 0.5-0.6 $\mathrm{r}_{180}$. Is is rather interesting that XMM-Newton, with an effective area more than ten times larger than ASCA or BeppoSAX is not capable of extending temperature measurements beyond regions already explored by the latter satellites. It is also a matter of some concern that the first comparisons between the XMM-Newton and BeppoSAX temperature points to a non-negligible disagreement (e.g. De Grandi \& Molendi these proceedings).

Finally, BeppoSAX, ASCA and XMM-Newton measurements of the metal abundance extend out to about $0.4 \mathrm{r}_{180}$, (e.g. De Grandi \& Molendi 2001). This is indeed the most difficult observable to measure from low surface regions, at least with the current CCD resolution of a few percent.

Thus we are forced to conclude that current data do not go out to the virial radius, as a matter of fact they do not even come close to it. The answer to this section question is that we know nothing from direct $X$-ray observations of the extremely important region surrounding the virial radius.

\section{How difficult would it be to design experiments capable of investigating these regions?}

Having learned that current experiments are not sensitive to cluster outer regions we ask ourselves how difficult it would be to design experiments capable of investigating these regions. The answer to this question is that it would not be particularly difficult, no major technological breakthrough is required. Indeed the answer lies in keeping the background under control. Let us see how experiments could be optimized to do that.

\section{1. $N X B<C X B$}

The first thing to do is to reduce the non X-ray background (NXB) to values smaller than the Cosmic X-ray background (CXB) in the whole energy band covered by our experiment. Given the greater difficulty in focusing higher energy X-rays and the flatness of the NXB spectrum this typically means having NXB $<$ CXB at the highest energies. It is worth remarking that none of the imaging experiments sensitive in the medium energy band $(2-10 \mathrm{keV})$, flown so far, operate in the NXB $<$ CXB regime. Neither the first generation experiments (BeppoSAX, ASCA), nor the second generation experiments (Chandra, XMM-Newton). As a matter of fact, the CXB/NXB ratio for the latter is worse than for the former and this is why XMM-Newton temperature profiles do not extend further out than ASCA or Beppo-SAX temperature profiles.

Achieving the NXB $<$ CXB requirement would allow us to go about a factor 10 fainter in surface brightness. This is not really impossible: in the case of XMM-Newton for example, simply placing the satellite in an equatorial Low Earth Orbit would do more 


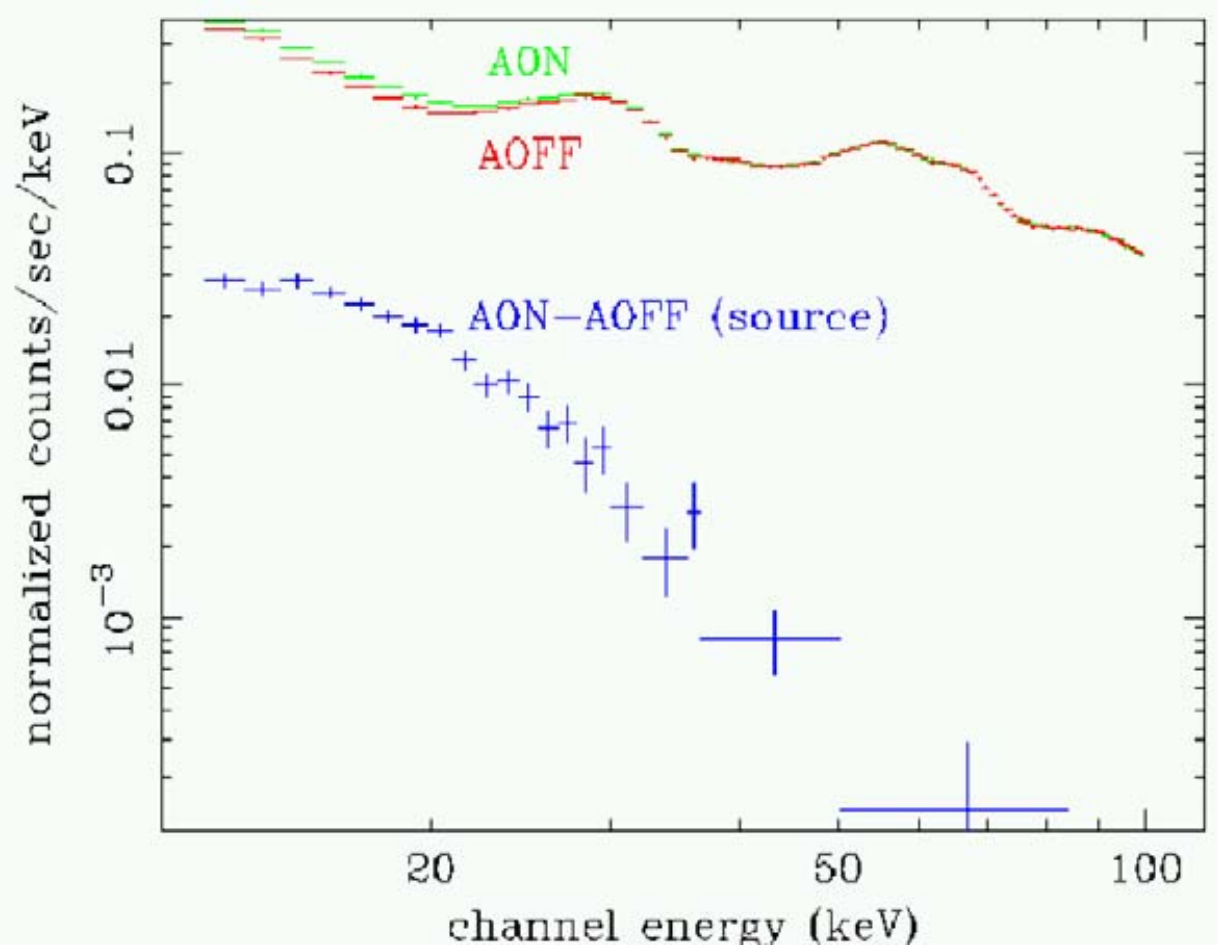

Figure 1. ON-spectrum, OFF-spectrum and source spectrum (difference between ON and $\mathrm{OFF}$ ) for collimator row A during a PDS pointing.

than half of the job. The rest, a factor of about 2, could probably be achieved by working on the shielding of the detectors.

\subsection{Observing strategy}

The other thing to do is to improve the observing strategy. Typically temperature measurements can be made out to radii where the source counts, cts $_{\text {sou }}$, are equal to the

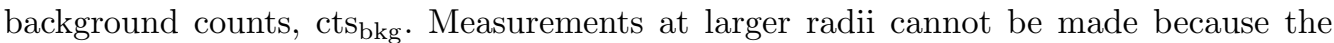
background is only poorly known. In other words we need to improve measurements in the cts $_{\text {sou }}<$ cts $_{\text {bkg }}$. This is where differential techniques can be very effective. The most striking example comes from radio wavelengths, indeed WMAP detects CMB anisotropies of 1 part in $10^{6}$ ! In the X-ray band the most successful experiment is the BeppoSAX PDS. This experiment makes use of 2 rows of rocking collimators: one is trained on the source, the other on the background; the collimators are switched every $\sim 100$ s to allow the detectors, viewed by both collimators, to observe source and background fields.

In Fig. 1 we show the ON-spectrum, the OFF-spectrum and the source spectrum (difference between $\mathrm{ON}$ and $\mathrm{OFF}$ ) for collimator row A during a PDS pointing. Extensive analysis (e.g. Rossetti 2003, Rossetti \& Molendi 2004) have shown that the source spectrum measurement is reliable down to where source counts are about $1 \%$ of the background counts. The strategy adopted by the PDS could easily be implemented in future imaging missions, simply by substituting collimators with telescopes.

Combining the two strategies (i.e. reducing the NXB intensity below the CXB intensity and using differential measurements to keep the background under control) we could 
easily go down by a factor of 100 in surface brightness reaching the required sensitivity to map outer regions of galaxy clusters.

\section{What about already planned missions?}

What about already planned X-ray missions? Will they do better than current ones in investigating cluster outskirts? Probably not, missions such as ASTRO-E2, Con-X and XEUS are not optimized for the study of low surface brightness emission. The general approach is to achieve a high sensitivity through a large effective area, paying relatively little attention to background issues. This works well for point-sources where the background can be kept under control through the size of the PSF, but is of limited use for extended sources, where the instrumental background intensity per unit solid angle increases with the telescope focal length.

Of course we are not trying to imply that planned missions will be useless for galaxy clusters. They will certainly allow us to study important cluster properties; as an example consider the measurements of gas motions and turbulence that will be possible with ASTRO-E2 (e.g. Sunyaev these proceedings). However these studies will be limited to the brighter regions of galaxy clusters. In a sense our investigations will be restricted to the tip of the proverbial iceberg.

\section{Summary}

Observational characterization of outer regions is a fundamental step in understanding clusters. Unfortunately current and planned experiments will not allow us to study these regions. Fortunately no major technological breakthroughs are required to design an experiment sensitive to cluster outskirts. Whether and when such an experiment will be designed and flown will depend upon many factors. One of the most important will be the awareness of the cluster community of the need for such a mission and its determination at supporting it.

\section{References}

Borgani, S., Murante, G., Springel, et al. 2004, MNRAS, 348, 1078.

De Grandi S., \& Molendi, S. these proceedings.

De Grandi, S. \& Molendi S. 2002, ApJ, 567, 163.

De Grandi, S. \& Molendi S. 2001, ApJ, 551, 153.

Ettori, S., Tozzi, P., Borgani, S., Rosati, P. 2004, A\&A, 417, 13.

Ettori, S., Fabian, A. C. 1999, MNRAS, 305, 834.

Markevitch, M., Forman, W. R., Sarazin, C. L., \& Vikhlinin, A. 1998, ApJ, 503, 77.

Mohr, J.J., Mathiesen, B., Evrard, A.E. 1999, ApJ, 517, 627.

Moore, B. these proceedings.

Pratt, G. W., Arnaud, M. 2002 A\&A, 394, 375.

Rossetti, M., Molendi, S 2004, A\&A, 414, L41.

Rossetti, M. Laurea Thesis 2003, Milano.

Schuecker, P., Finoguenov, A., Miniati, F., Boehringer, H., Briel, U.G. 2004, A\&A sub. astro$\mathrm{ph} / 0404132$.

Sunyaev, R. these proceedings.

Tozzi, P., Scharf, C. \& Norman, C. 2000, ApJ, 542, 106.

Treu, T. these proceedings.

Vikhlinin, A., Markevitch, M., Forman, W., Jones, C 2001, ApJ, 555, L87.

Zhang, Y.-Y., Finoguenov, A., Boehringer, et al. 2004, A\&A, 413, 49. 